Supplementary Information for

\title{
Hybrid Metallic Foam with Superior Elasticity, High Electrical \\ Conductivity and Pressure Sensitivity
}

Yan Peng, ${ }^{\text {a }}$ Huaizhi Liu, ${ }^{\text {a }}$ Tuoqi Li,${ }^{b}$ Jiuyang Zhang ${ }^{a *}$

a School of Chemistry and Chemical Engineering, Southeast University, Nanjing, 211189, PR China; Jiangsu Hi-Tech Key Laboratory for Biomedical Research, 211189, Nanjing, PR China

b Department of Chemical Engineering and Materials Science, University of Minnesota, Minneapolis, Minnesota 55455, United States

Corresponding Author

*E-mail: jiuyang@seu.edu.cn 


\section{Experimental Section}

Instrumentation: Biosafer 150-96 ultrasonic crasher was utilized to disperse LM in MDI and DCM. Samples were sonicated for 5 minutes, power at $45 \%$. Field Emission Scanning Electron Microscope (FE-SEM), Energy-dispersive X-ray spectroscopy (EDS) and optical microscope were used to characterize the morphology of LM-Foams. The LM-Foams for SEM and EDS were first frozen in liquid nitrogen and then split into small pieces immediately. The porous structures of foams were observed in SEM (FEI 3D). The pure polyurethane and LM-Foams for optical microscope were first frozen in liquid nitrogen and then cut into cylinders immediately. The porous structures of foams and coated LM were observed in optical microscope. True density analyzer AccuPyc 1330 was used to obtain true density and calculate porosity. Mechanical measurement: Samples for tensile and compressive tests were long bars prepared in needle and cylinders foamed in mold with diameter of $4.7 \pm 0.1 \mathrm{~mm}$ and $17.6 \pm 0.4 \mathrm{~mm}$, respectively. Mechanical (tensile and compressive) tests were carried out on an SANS E42.503 tensile tester at room temperature with a speed of $15 \mathrm{~mm} / \mathrm{min}$ except cyclic test which was $60 \mathrm{~mm} / \mathrm{min}$. The fixture can be removed depending on the type of tests and samples. All reported values for mechanical properties were an average of at least three independent tests for each sample. Resistance measurement: The real-time resistance was monitored by the Keysight $34461 \mathrm{~A}$ at room temperature. In compressive test, copper adhesive (copper foil with adhesive in the back) was used to connect LM-Foam and Keysight 34461A and polytetrafluoroethylene (PTFE) film was acted as insulator. The number of power 
line cycles (NPLC) was 0.02 and measurement range was automatic mode. The compressive samples $(\Phi=17.6 \pm 0.4 \mathrm{~mm}, \mathrm{~L}=10 \pm 1 \mathrm{~mm})$ were placed between two fixtures covered by PTFE film, and copper adhesive was stick on the PTFE film to connected LM-Foam to Keysight 34461A. In handwriting tests, the LM-Foam $(\Phi=$ $17.6 \pm 0.4 \mathrm{~mm}, \mathrm{~L}=2.5 \pm 0.1 \mathrm{~mm}$ ) was placed on petri dish with copper adhesive and covered by PVC film. Copper adhesive was stuck on both side of LM-Foam to connect to Keysight 34461A. For balls and truck collision test, LM-Foam was fixed by PVC film Copper adhesive was stuck on both side of LM-Foam to connect to Keysight 34461A.

Preparation of pure polyurethane: PTTO (0.5 g) and MDI (0.2 g) were added dropwise and mixed at a ratio of 5: 2 by weight in the cylinder mold. The mixture was stirred for 15 seconds and polyurethane was obtained in 2 minutes. The obtained polyurethane foam was a white cylinder.

Preparation of LM-Foams: 1) A certain volume of liquid metal was mixed with $25 \mathrm{wt} \% \mathrm{MDI}(0.05 \mathrm{~g})$ in $5 \mathrm{~mL}$ glass bottle. Then added DCM $(1.5 \mathrm{~mL})$ and controlled the concentration of MDI to $0.03 \mathrm{~g} / \mathrm{mL}$. The mixture was ultrasonicated for 5 minutes at room temperature by Biosafer 150-96 ultrasonic crasher until DCM was basically volatilized. 2) The remaining $75 \mathrm{wt} \%$ MDI $(0.15 \mathrm{~g})$ was added dropwise to the obtained mixture of 1) after they had been transferred to the cylinder mold. Then PTTO (0.5 g) was added dropwise at a ratio of 5: 2 to MDI by weight. LM-Foam can be obtained by 15 seconds of stirring and 2 minutes of standing at room temperature. The obtained LM-Foams were white gray cylinders. The process can be found in 


\section{Video S1.}

Preparation of CB-Foams: 1) A certain weight of carbon black was mixed with $25 \mathrm{wt} \%$ MDI $(0.05 \mathrm{~g})$ in $5 \mathrm{~mL}$ glass bottle. Then added DCM $(1.5 \mathrm{~mL})$ and controlled the concentration of MDI to $7.5 \mathrm{mg} / \mathrm{mL}$. The mixture was ultrasonicated for 5 minutes at room temperature by Biosafer 150-96 ultrasonic crasher until DCM was basically volatilized. 2) The remaining $75 \mathrm{wt} \%$ MDI $(0.15 \mathrm{~g})$ was added to the obtained mixture of 1) after they had been transferred to the cylinder mold. Then PTTO (0.5 g) was added at a ratio of 5: 2 to MDI by weight. CB-Foam can be obtained by 15 seconds of stirring and 2 minutes of standing at room temperature. The obtained CB-Foams were black cylinders.

\section{Preparation of Poly(hydroxyethyl acrylate)-Polyacrylamide (PHEA-PAM)}

hydrogel: $1.4 \mathrm{~g} \mathrm{AM}$ and $0.6 \mathrm{~g}$ HEA were dissolved in $6 \mathrm{~mL}$ distilled water in $20 \mathrm{~mL}$ glass bottle. Then, $20 \mathrm{mg}$ APS and $10 \mu \mathrm{L}$ TEMED were added into the solution and mixed evenly to initiate and catalyze the gelation. The PHEA-PAM hydrogel was obtained at room temperature for 15 minutes. The obtained PHEA-PAM hydrogel was transparent cylinder. 


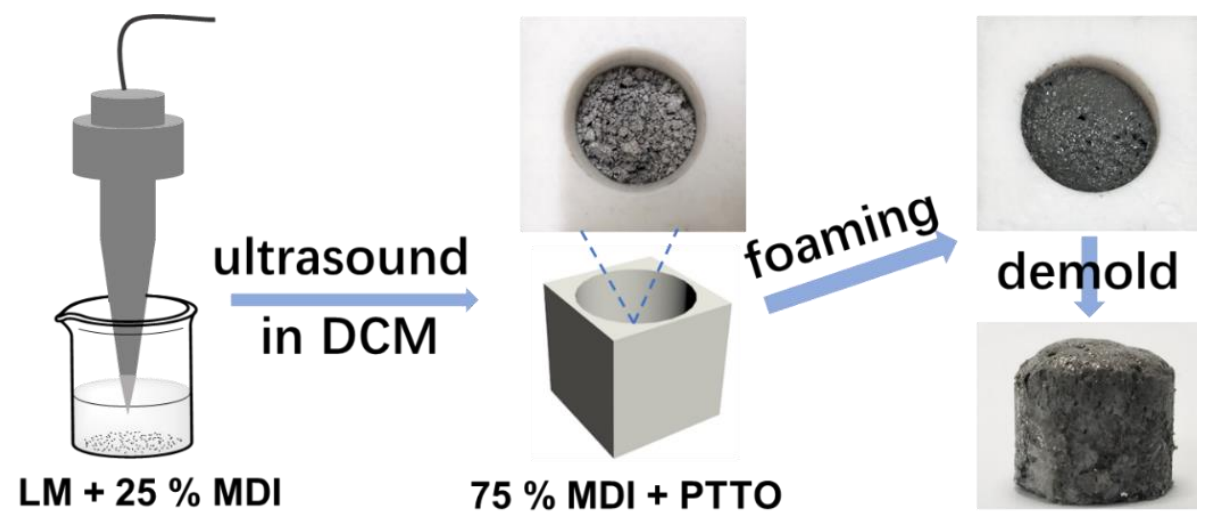

Figure S1. Scheme for the preparation of LM-Foams. LM and $25 \mathrm{wt} \%$ MDI were dispersed in DCM by sonication and then transferred to the cylinder mold. The remaining $75 \mathrm{wt} \%$ MDI and PTTO was added dropwise to the mold. LM-Foam can be obtained by 15 seconds of stirring and 2 minutes of standing at room temperature. The process can be found in Video S1.

a

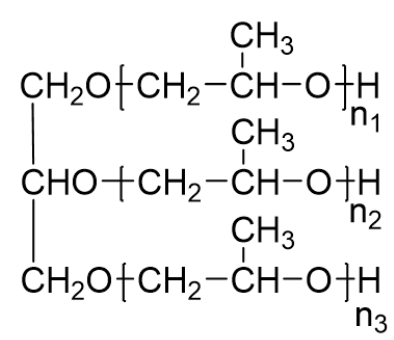<smiles>O=Nc1ccc(Cc2ccc(N=O)cc2)cc1</smiles>

MDI

PTTO

b $\mathrm{O}=\mathrm{C}=\mathrm{N}-\mathrm{R}_{1}-\mathrm{N}=\mathrm{C}=\mathrm{O}+\mathrm{HO}-\mathrm{R}_{2}-\mathrm{OH}+$ Diisocyanate(DI) Polyether polyol(PPO)

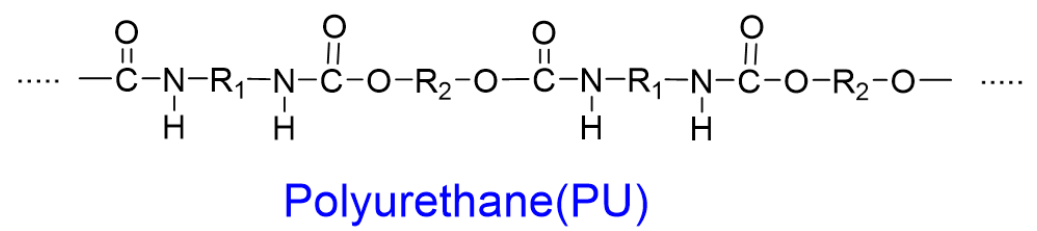

Figure S2. (a) Chemical structure of PTTO and MDI. (b) The reaction equation of diisocyanate and polyether polyol to synthesize polyurethane. 
Table S1. Detail information of LM-Foams, including the weight ratio of MDI, PTTO and volume of DCM and LM.

\begin{tabular}{ccccc}
\hline Samples & MDI & PTTO & DCM & $\begin{array}{c}\text { LM } \\
\text { (melting point }=\mathbf{1 0}{ }^{\circ} \mathbf{C} \text { ) }\end{array}$ \\
\hline LM-Foam-0\% a & $0.5 \mathrm{~g}$ & $0.2 \mathrm{~g}$ & $1.5 \mathrm{~mL}$ & $0 \mu \mathrm{L}$ \\
LM-Foam-10\% & $0.5 \mathrm{~g}$ & $0.2 \mathrm{~g}$ & $1.5 \mathrm{~mL}$ & $66 \mu \mathrm{L}$ \\
LM-Foam-20\% & $0.5 \mathrm{~g}$ & $0.2 \mathrm{~g}$ & $1.5 \mathrm{~mL}$ & $150 \mu \mathrm{L}$ \\
LM-Foam-30\% & $0.5 \mathrm{~g}$ & $0.2 \mathrm{~g}$ & $1.5 \mathrm{~mL}$ & $255 \mu \mathrm{L}$ \\
LM-Foam-40\% & $0.5 \mathrm{~g}$ & $0.2 \mathrm{~g}$ & $1.5 \mathrm{~mL}$ & $395 \mu \mathrm{L}$ \\
LM-Foam-50\% & $0.5 \mathrm{~g}$ & $0.2 \mathrm{~g}$ & $1.5 \mathrm{~mL}$ & $595 \mu \mathrm{L}$ \\
\hline
\end{tabular}

a The percentage indicated the volume fraction of LM in feeding ratio, including MDI, PTTO and LM. $\left(\rho(\mathrm{MDI})=1.05 \mathrm{~g} / \mathrm{cm}^{3} ; \rho(\mathrm{PTTO})=1.24 \mathrm{~g} / \mathrm{cm}^{3}\right)$ 

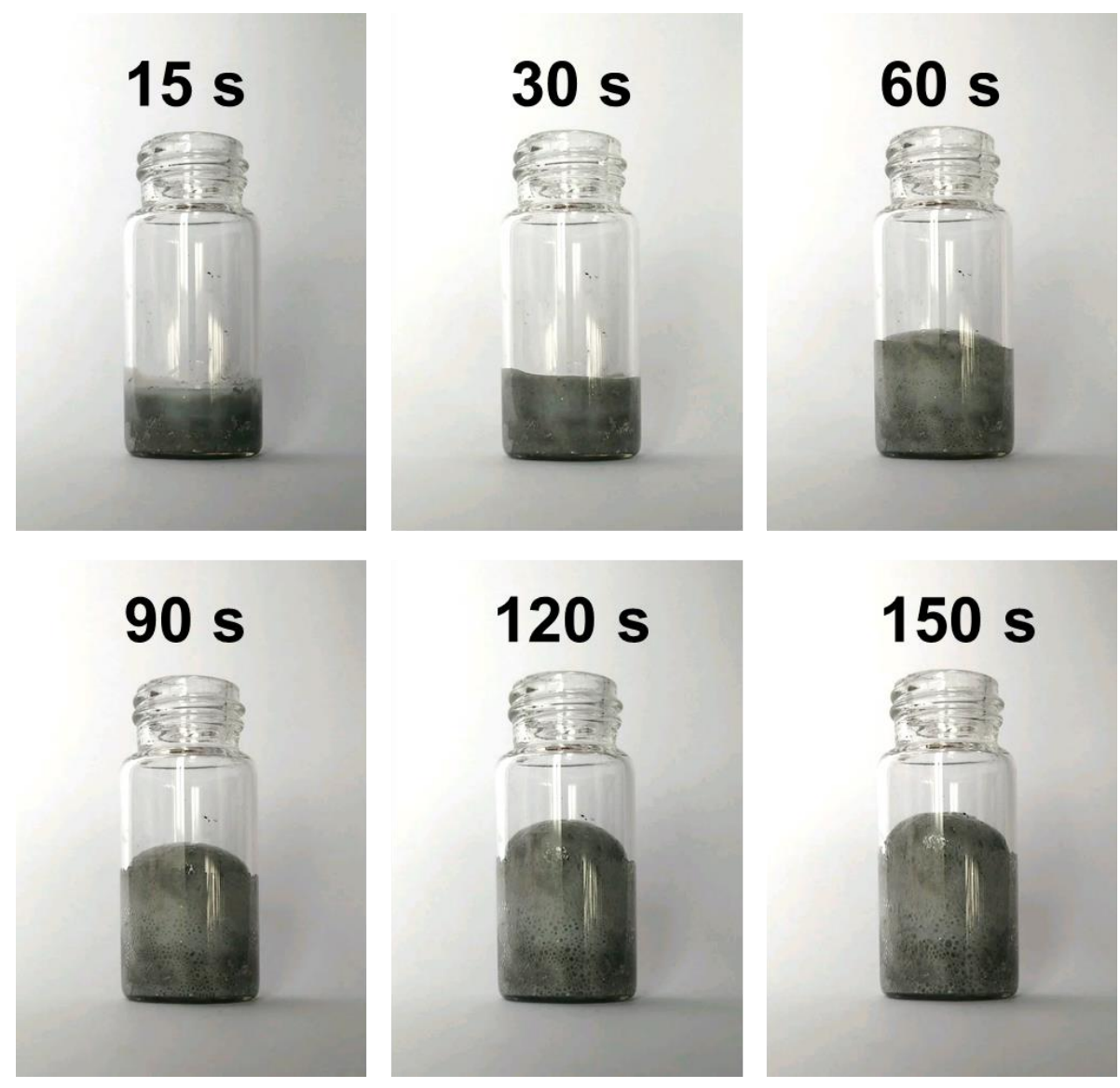

Figure S3. Photos of foaming process at different time. The viscosity of mixture increased sharply while stirring and began foaming within $20 \mathrm{~s}$. The reaction was completed in approximately 2 minutes. The process was shown in video S1. 

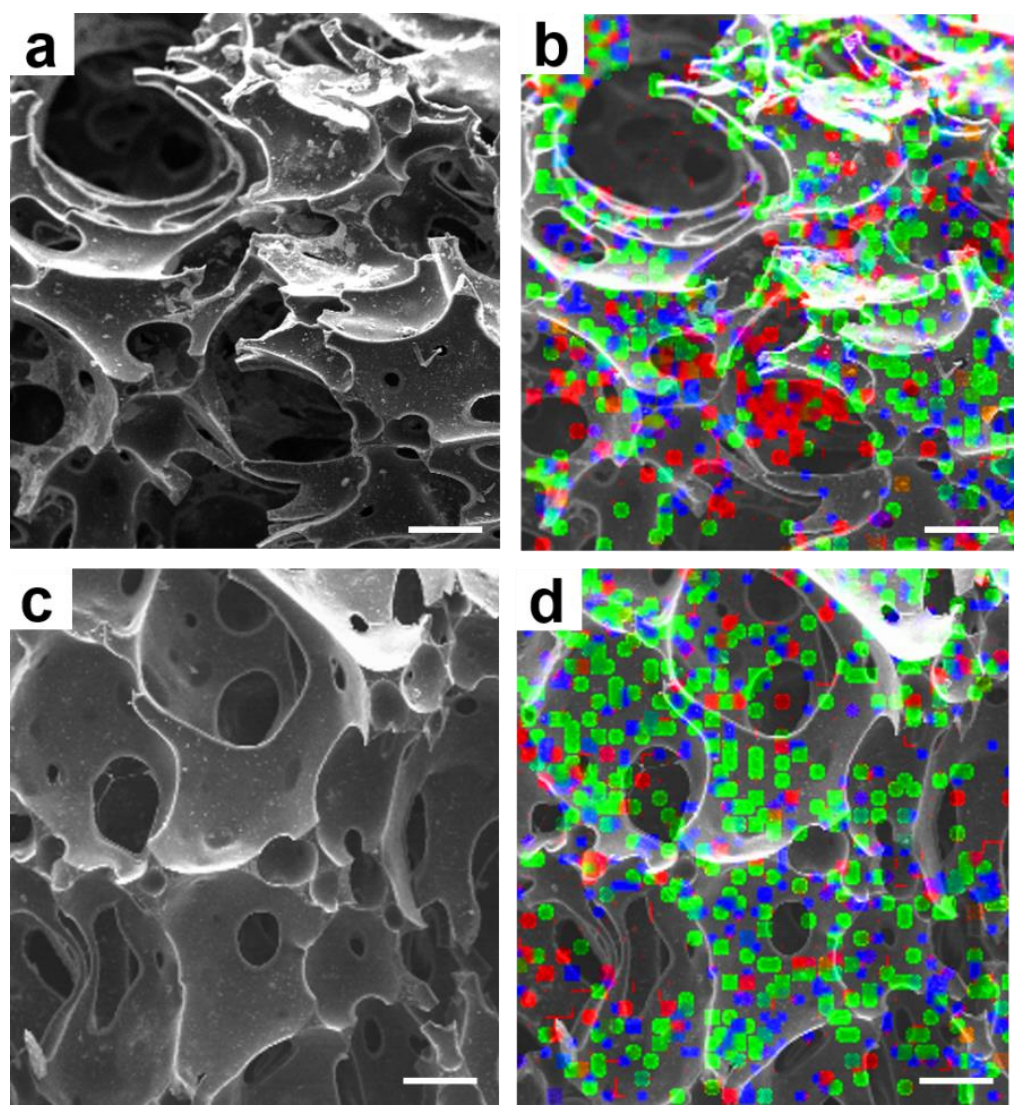

Figure S4. (a) Cross-sectional scanning electron microscope (SEM) and (b) Energy-dispersive X-ray spectroscopy (EDS) image of LM-Foam-30\%; (c) Cross-sectional scanning electron microscope (SEM) and (d) Energy-dispersive X-ray spectroscopy (EDS) image of LM-Foam-40\%. (Green, red and blue represent Ga, Indium and Sn, respectively.) Scale bar: $200 \mu \mathrm{m}$.

Table S2. EDS composition analysis of LM-Foam-40\%, including weight fraction and atomic fraction.

\begin{tabular}{ccc}
\hline Element & Weight \% & Atomic \% \\
\hline Ga & 76.77 & 84.68 \\
In & 12.23 & 8.18 \\
Sn & 10.99 & 7.12 \\
\hline
\end{tabular}




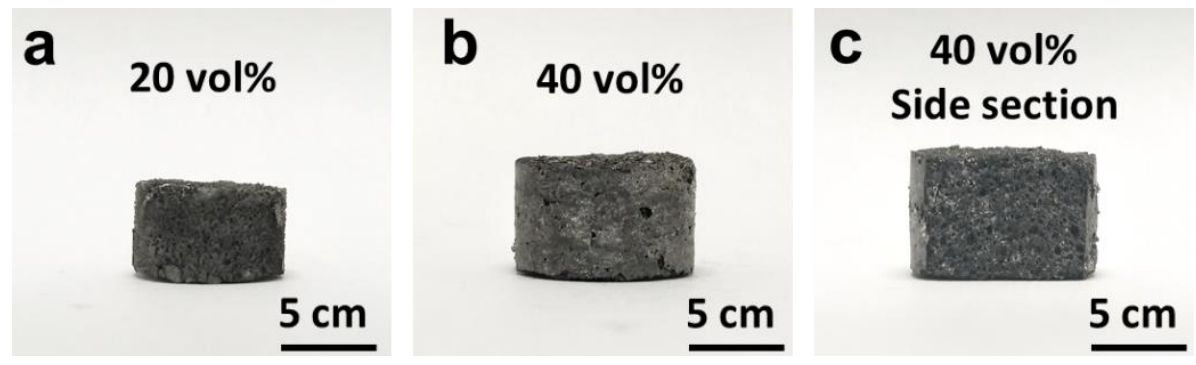

Figure S5. Photos of LM-Foam-20\% (a), LM-Foam-40\% (b) and side section of LM-Foam-40\% (c). Scale bar: $5 \mathrm{~cm}$.
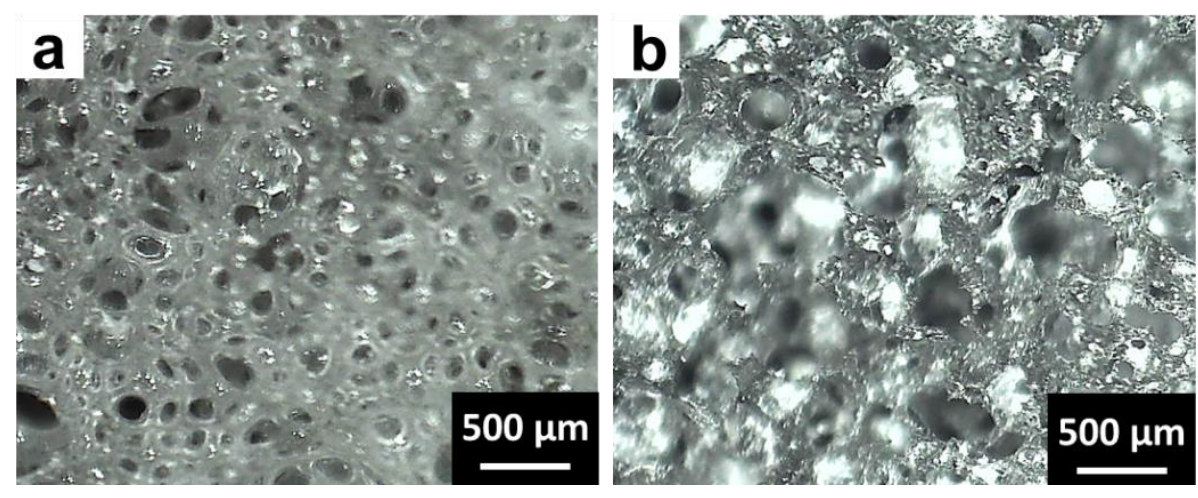

Figure S6. Cross-sectional optical microscopy pictures of LM-Foam-20\% (a) and LM-Foam-40\% (b). Scale bar: $500 \mu \mathrm{m}$.

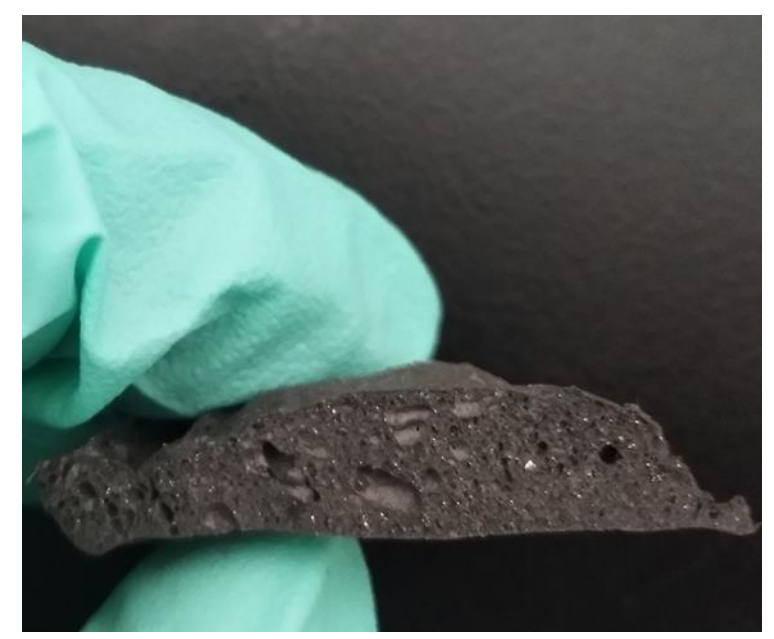

Figure S7. Photo of LM-Foam-20\% (The melting point of liquid metal is $58{ }^{\circ} \mathrm{C}$ ) 

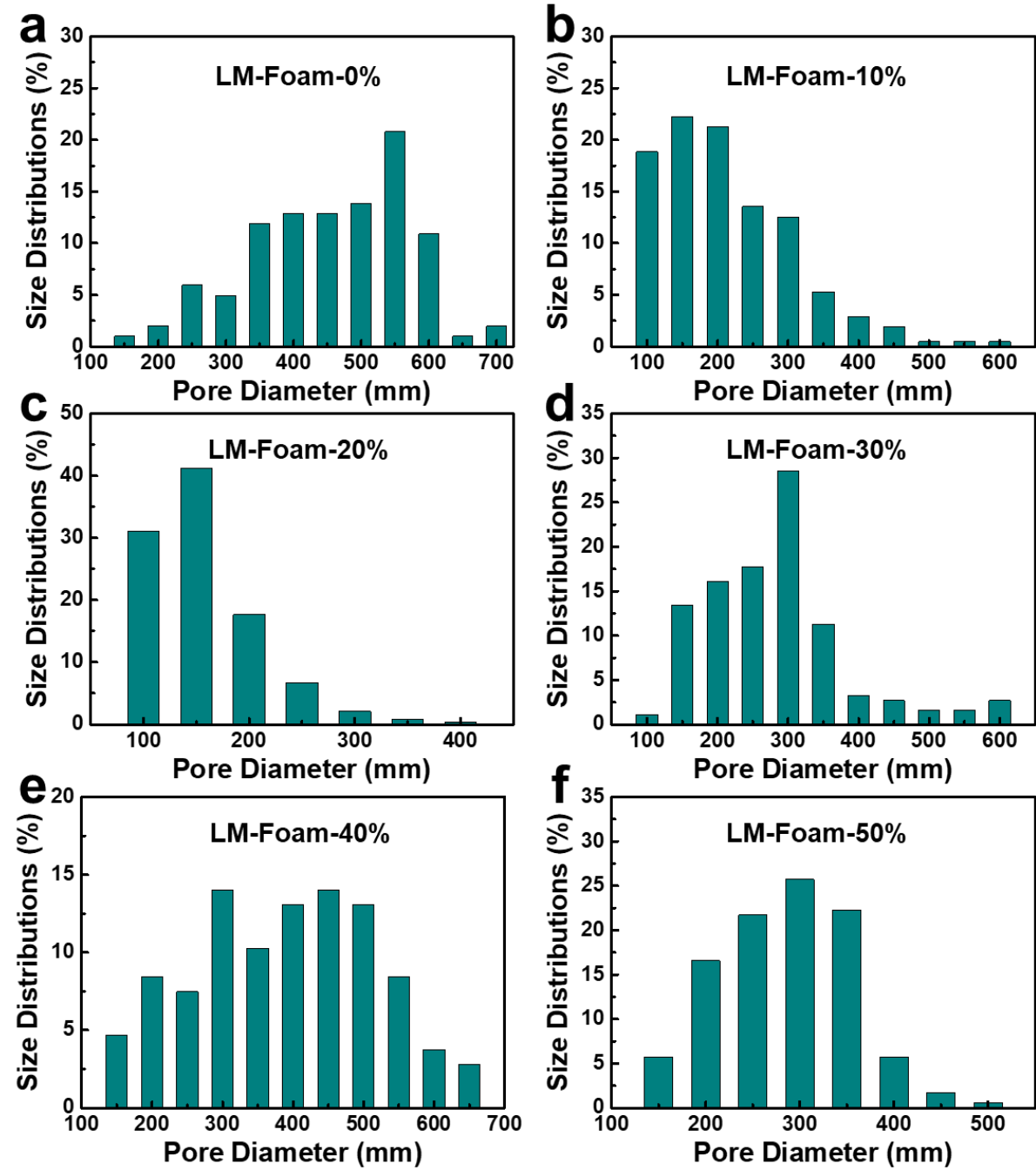

Figure S8. Pore size distributions of LM-Foams with different LM content. 


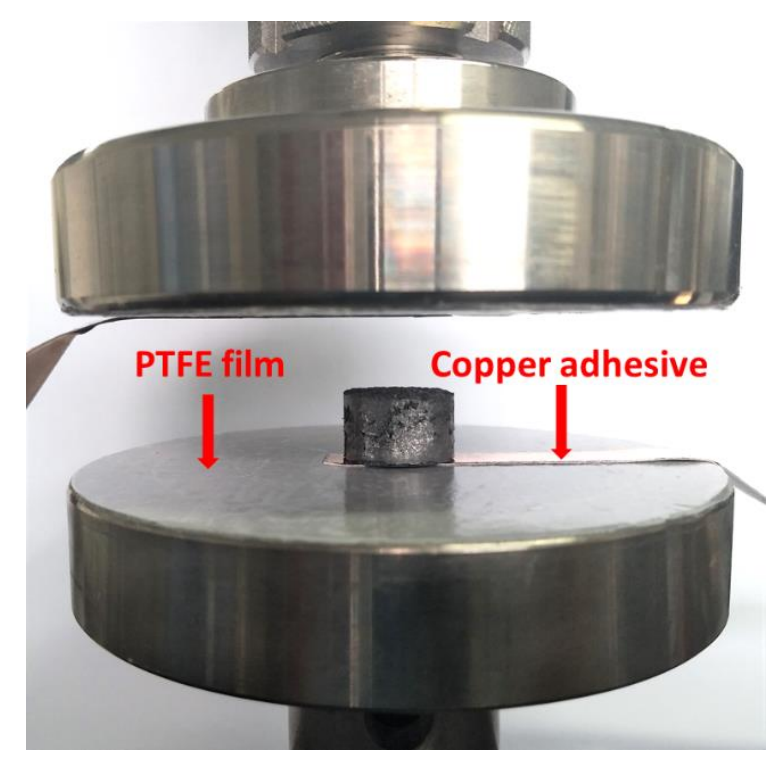

Figure S9. Experimental setup of compressive test and electrical measurement. The Polytetrafluoroethylene (PTFE) was used as an insulator. Copper adhesive was utilized to connect LM-Foam to Keysight 34461A.

Table S3. The mechanical properties of LM-Foams in compressive test.

\begin{tabular}{ccc}
\hline Samples & ${\boldsymbol{\sigma ~}(\mathbf{k P a})^{\mathbf{a}}}$ & Uhys coefficient $(\%)^{\mathbf{b}}$ \\
\hline LM-Foam-0\% & $530 \pm 7$ & 27.4 \\
LM-Foam-10\% & $270 \pm 5$ & 32.1 \\
LM-Foam-20\% & $528 \pm 3$ & 37.4 \\
LM-Foam-30\% & $657 \pm 17$ & 36.1 \\
LM-Foam-40\% & $725 \pm 6$ & 37.0 \\
LM-Foam-50\% & $929 \pm 18$ & 42.0 \\
\hline
\end{tabular}

${ }^{a}$ The maximum compressive stress of LM-Foams at a strain of $95 \%$. ${ }^{b}$ The energy dissipation coefficient ( $U_{h y s}$ coefficient) defined as the ratio of dissipated energy (area circled by one mechanical test cycle) versus compression curve part. 


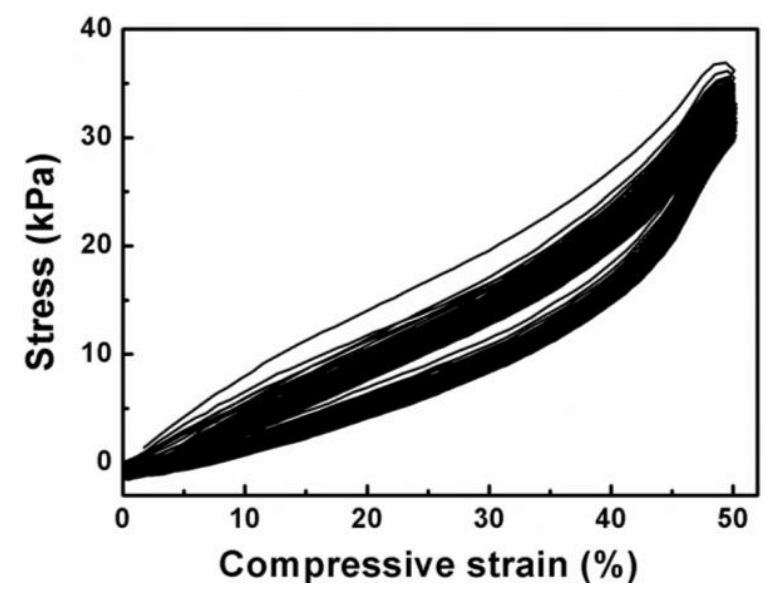

Figure S10. Cyclic compression-release test (1000 cycles) of pure polyurethane at strain of $50 \%$.

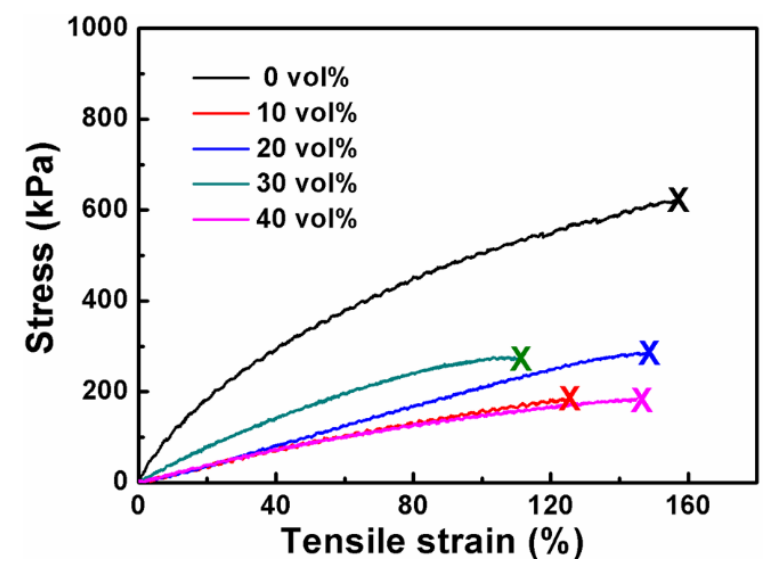

Figure S11. Tensile stress-strain curves of LM-Foams. The samples were prepared by injecting the mixture into needle tubes. 


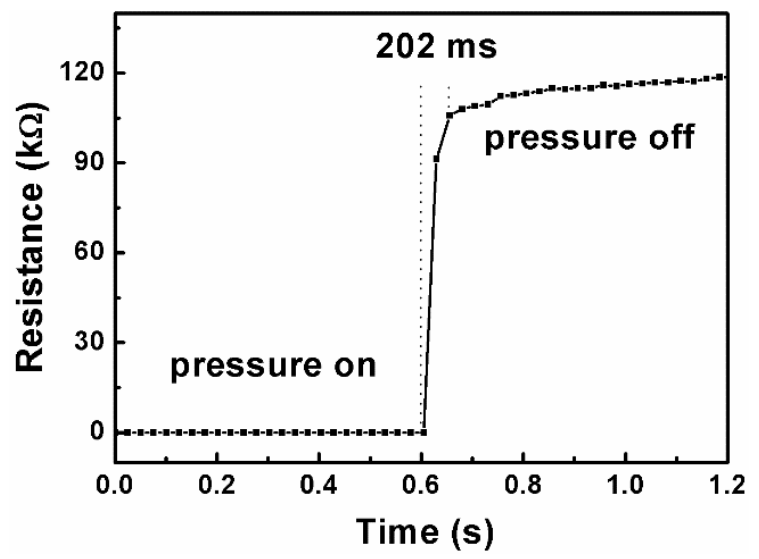

Figure S12. The responding time of the LM-Foam-40\% towards external pressure.

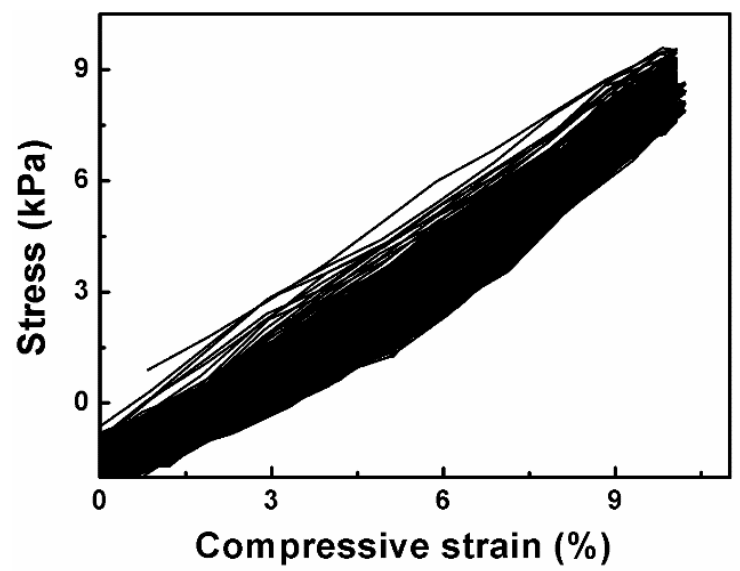

Figure S13. Compressive stress-strain curves of LM-Foam- $40 \%$ in the process of 800 cyclic compressive test at strain of $10 \%$. 
Table S4. Detail information of CB-Foams, including the weight ratio of MDI, PTTO and $\mathrm{CB}$.

\begin{tabular}{cccc}
\hline Samples & MDI & PTTO & CB $\left(\boldsymbol{\rho}=\mathbf{0 . 2 5} \mathbf{g} / \mathbf{c m}^{\mathbf{3}}\right)$ \\
\hline LM-Foam-10\% & $0.5 \mathrm{~g}$ & $0.2 \mathrm{~g}$ & $16 \mathrm{mg}$ \\
LM-Foam-20\% & $0.5 \mathrm{~g}$ & $0.2 \mathrm{~g}$ & $37 \mathrm{mg}$ \\
LM-Foam-30\% & $0.5 \mathrm{~g}$ & $0.2 \mathrm{~g}$ & $64 \mathrm{mg}$ \\
LM-Foam-40\% & $0.5 \mathrm{~g}$ & $0.2 \mathrm{~g}$ & $99 \mathrm{mg}$ \\
\hline
\end{tabular}

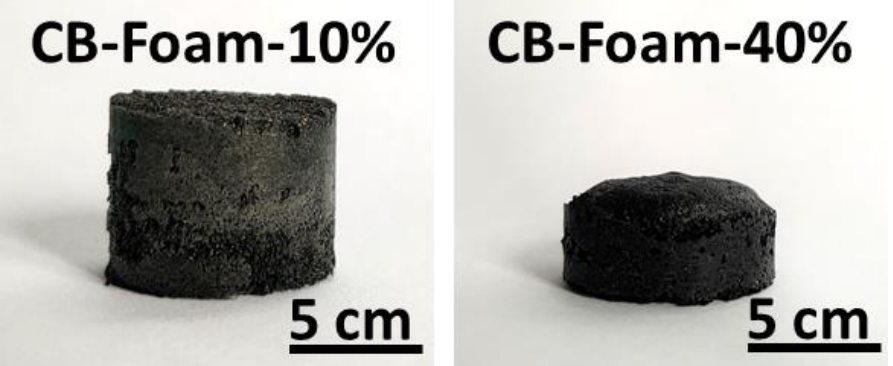

Figure S14. Photos of CB-Foam-10\% and CB-Foam-40\%. CB-Foam-10\%, 20\% and $30 \%$ were non-conductive. When the volume fraction increased to $40 \%$, the mixture cannot foam. Scale bar: $5 \mathrm{~cm}$.

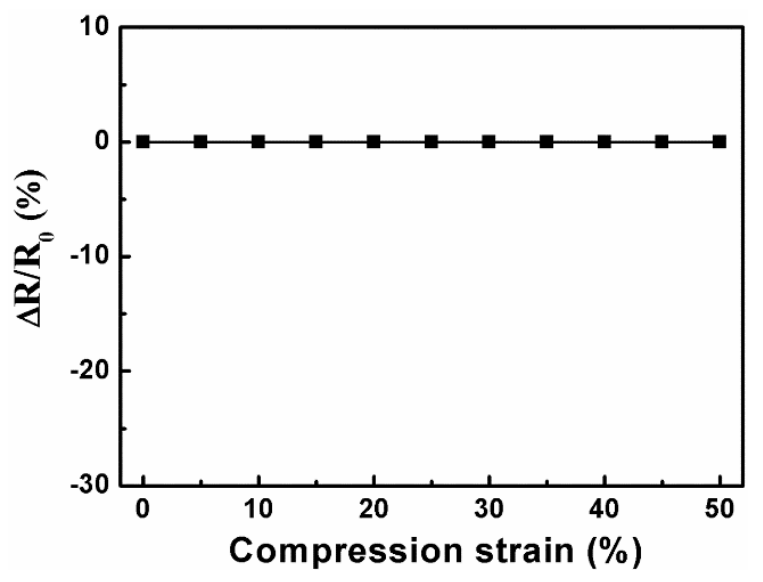

Figure S15. Curves of resistance change rate and compressive strain of CB-Foam-30\%. The sample cannot realize conductivity under stress.

Illustration for writing tests. 
The writing pad was fabricated as shown in Figure S16a and 16b. Photo for the pad device was shown in Figure S17. The pad was divided into nine areas (Figure S16b), and a compressive force $(2 \mathrm{kPa})$ was applied on different positions. Surprisingly, LM-Foam sensors showed strong position-dependent responses in Figure S16c, which was attributed by the non-uniform pore distribution in polymer matrix (SEM images in Figure 1) together with the deformable liquid fillers on pore surface. The increased and decreased resistance may be attributed by the connection and disconnection of LM droplets in foam matrix upon external shear and pressing force. Hand-writing tests were carried out by using a pen to apply continuous pressure in one direction on different positions of the pad surface. Different electrical signals were observed during the same writing at different regions (Figure S16d, 16e, and 16f, Video S3), demonstrating the inhomogeneous distribution of pores inside the foam matrix. To further confirm this viewpoint, a conductive hydrogel, poly(hydroxyethyl acrylate)-polyacrylamide (PHEA-PAM), was prepared as a homogeneous control to exclude the set-up effects on electrical signals. Interestingly, the PHEA-PAM hydrogel was incapable to exhibit various signals on different positions (Figure S18), again indicating the critical role of pore structures in foam. Attributed by the above unique sensory behaviors, LM-Foam was very promising as advanced writing and security pads to protect private information or property. As shown in Figure S16g and S19, different patterns of resistive change were observed for writing different letters (Figure S19) or even the same letters with different sizes (Figure S16g). Nearly the same resistive response pattern was produced when writing 
word "A" for three times, indicating the excellent electrical stability. This phenomenon allowed people to set their private passwords to unlock the smart devices. Interestingly, for the same word "OK" written by two different researchers (Figure S16h and S16i), the pad presented distinctive signals for two individuals, showing the potential to monitor personal writing habits. This feature offers double certification that even people disclose their passwords; the device still won't be unlocked unless the people write the words in person. In contrast, hydrogels (PHEA-PAM) were unable to discern different human writings due to homogeneous internal structures (Figure S20). 


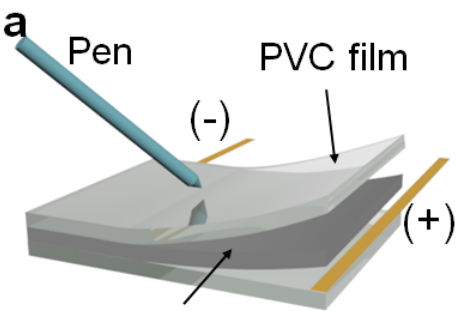

LM-Foam
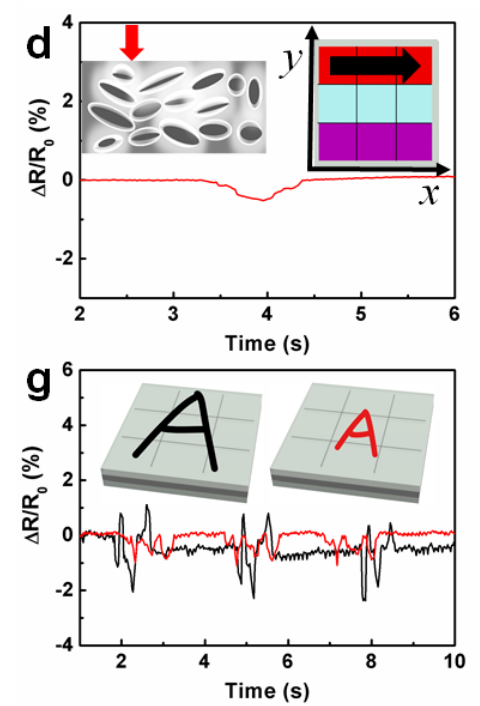

b
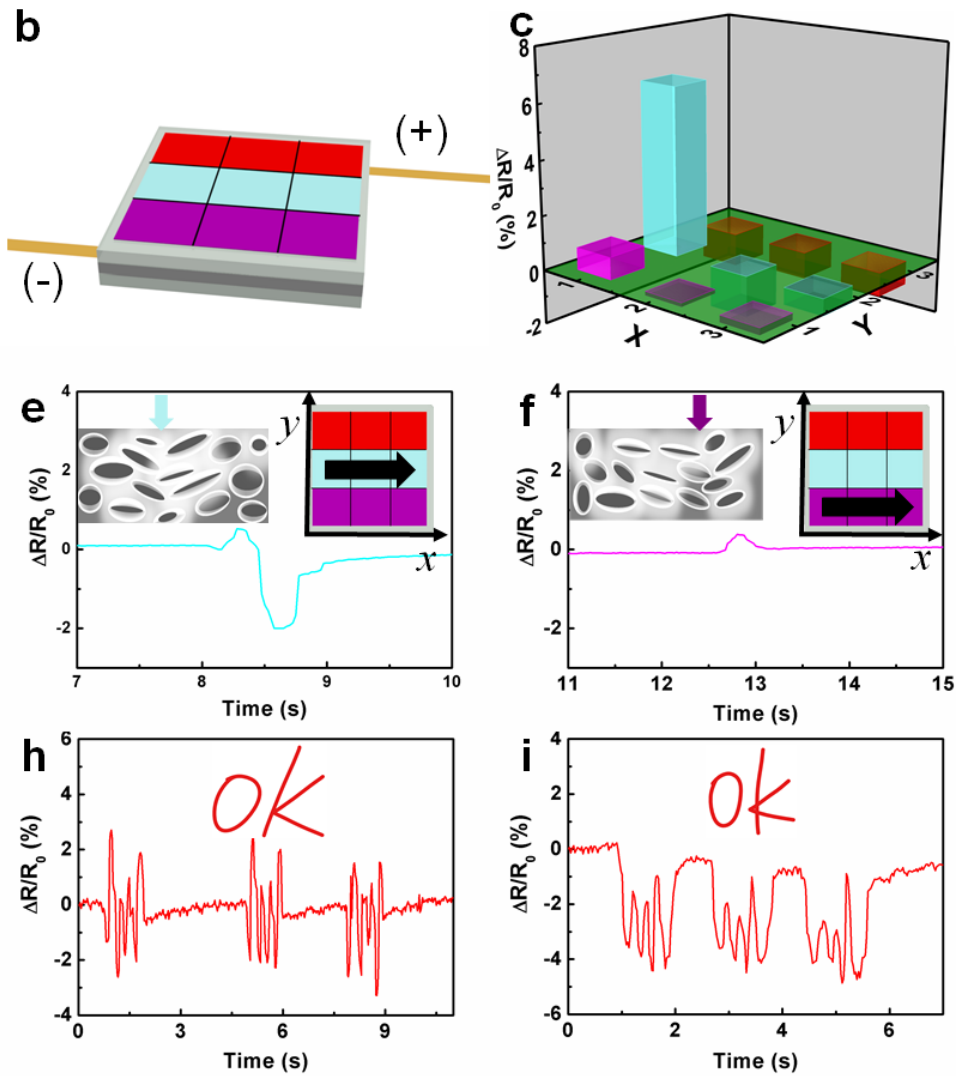
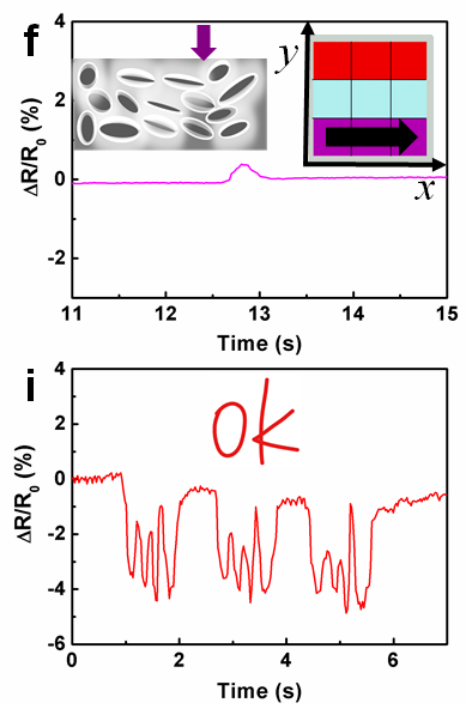

Figure S16. (a) and (b) Schematic illustration for the setup of password pads; (c)

Column graph of resistance change under compressive force of $2 \mathrm{kPa}$ at different positions the of the pressure pad; $\mathrm{X}$ and $\mathrm{Y}$ are coordinates of the pad surface as shown in Figure S16b; (d), (e) and (f) Resistive change of the pad under different writing path (Top, Middle and Bottom). The left insets showed the pore difference under the path of writing; The right insets showed the writing positions of the pad; (g) Electrical response of the writing on the pad when written different size of letter "A"; (h)-(i) “OK” written by different researchers. All devices were fabricated by LM-Foam- $40 \%$. 

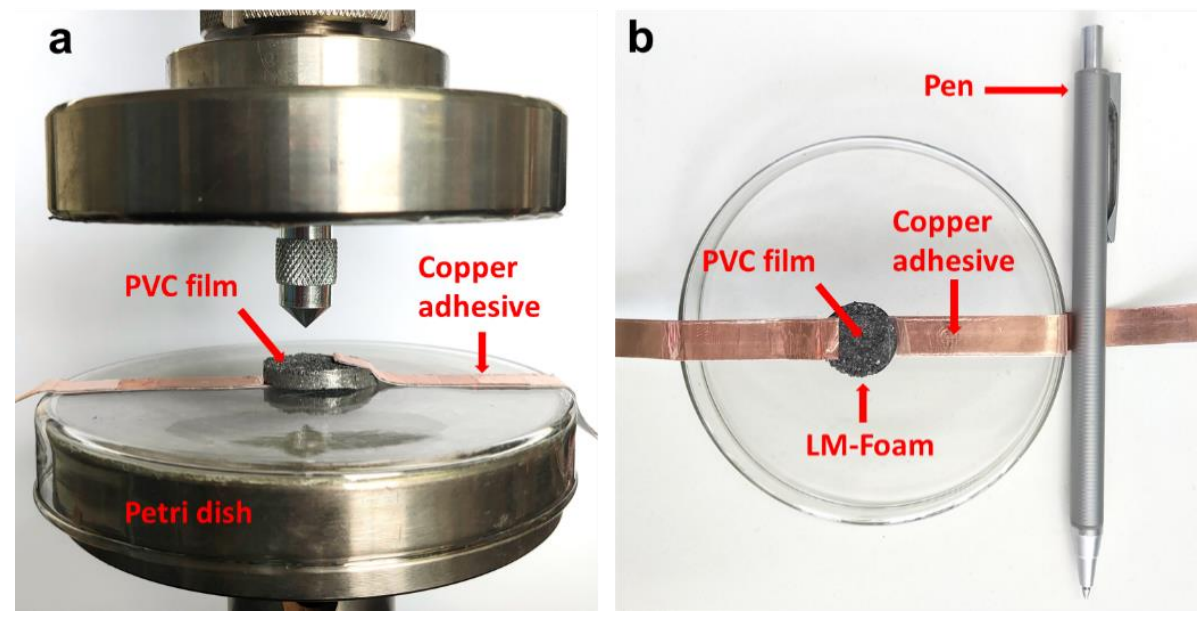

Figure S17. (a) and (b) Experimental setup of stabbing and handwriting pads.

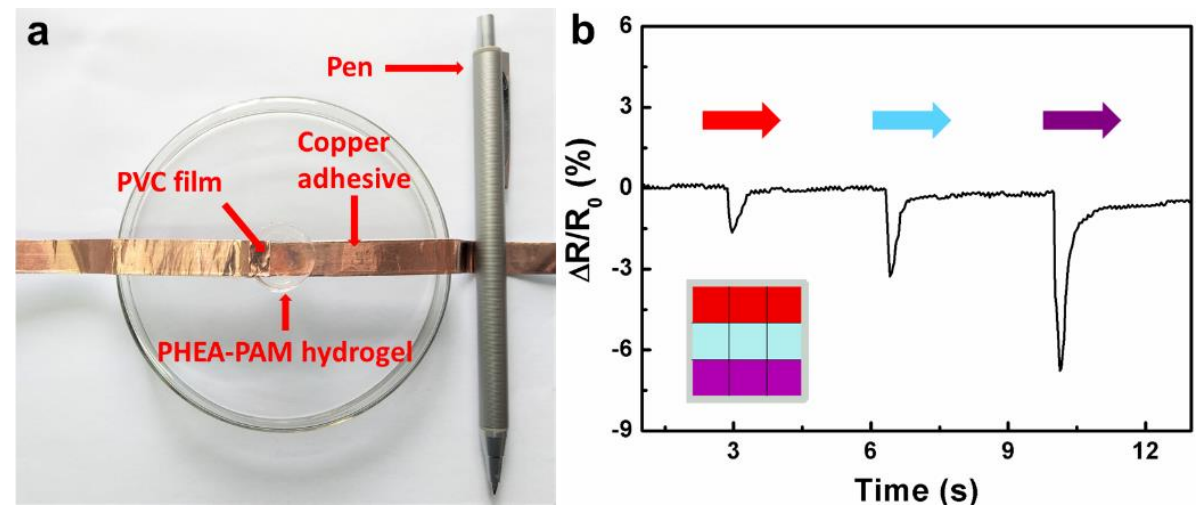

Figure S18. (a) The experimental setup of PHEA-PAM hydrogel handwriting test. The hydrogel is transparent. (b) Resistive change of PHEA-PAM hydrogel under different writing path. There were no significant differences in waveform. 

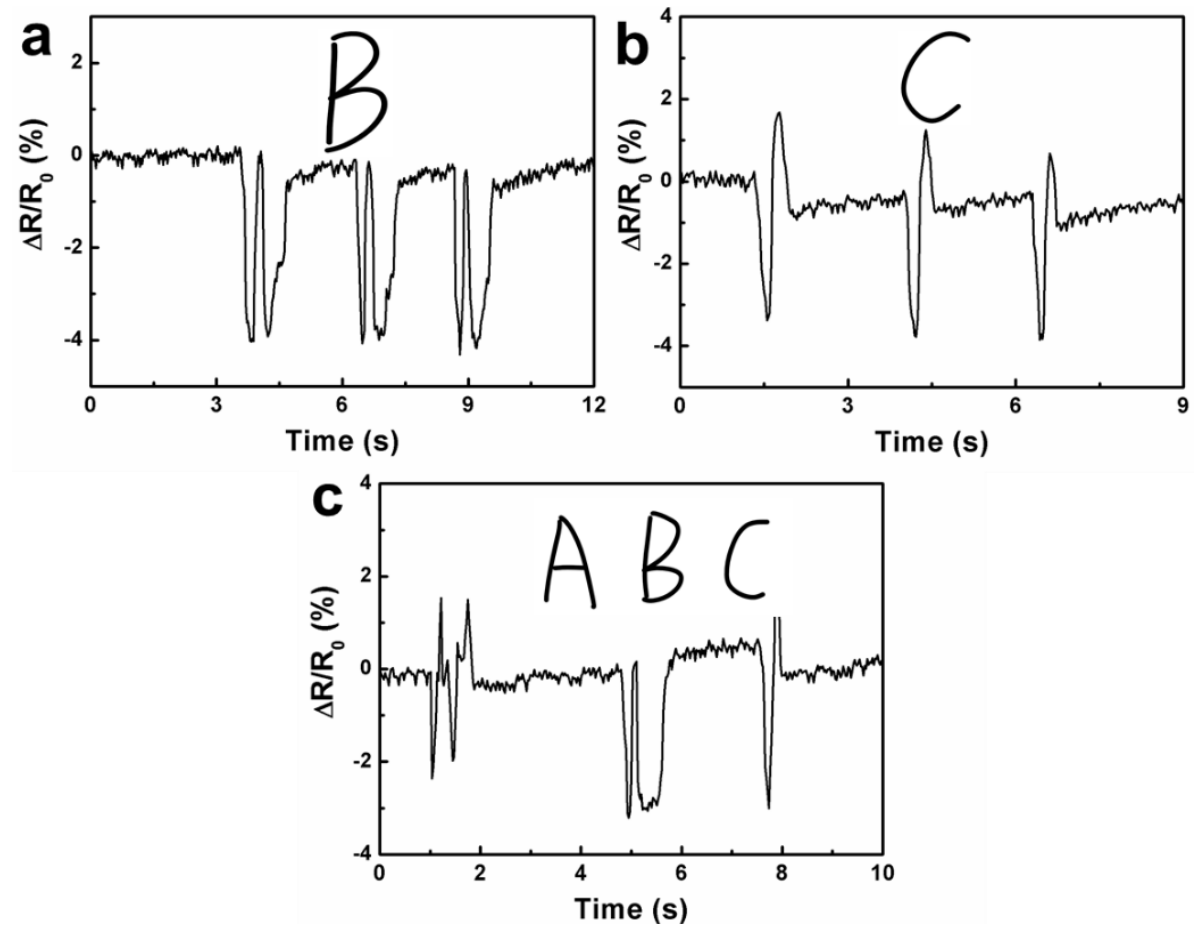

Figure S19. Electrical response of the writing on the pad when written letter "B" (a),

"C" (b) and continuous "ABC" (c). Device was fabricated by LM-Foam-40\%.
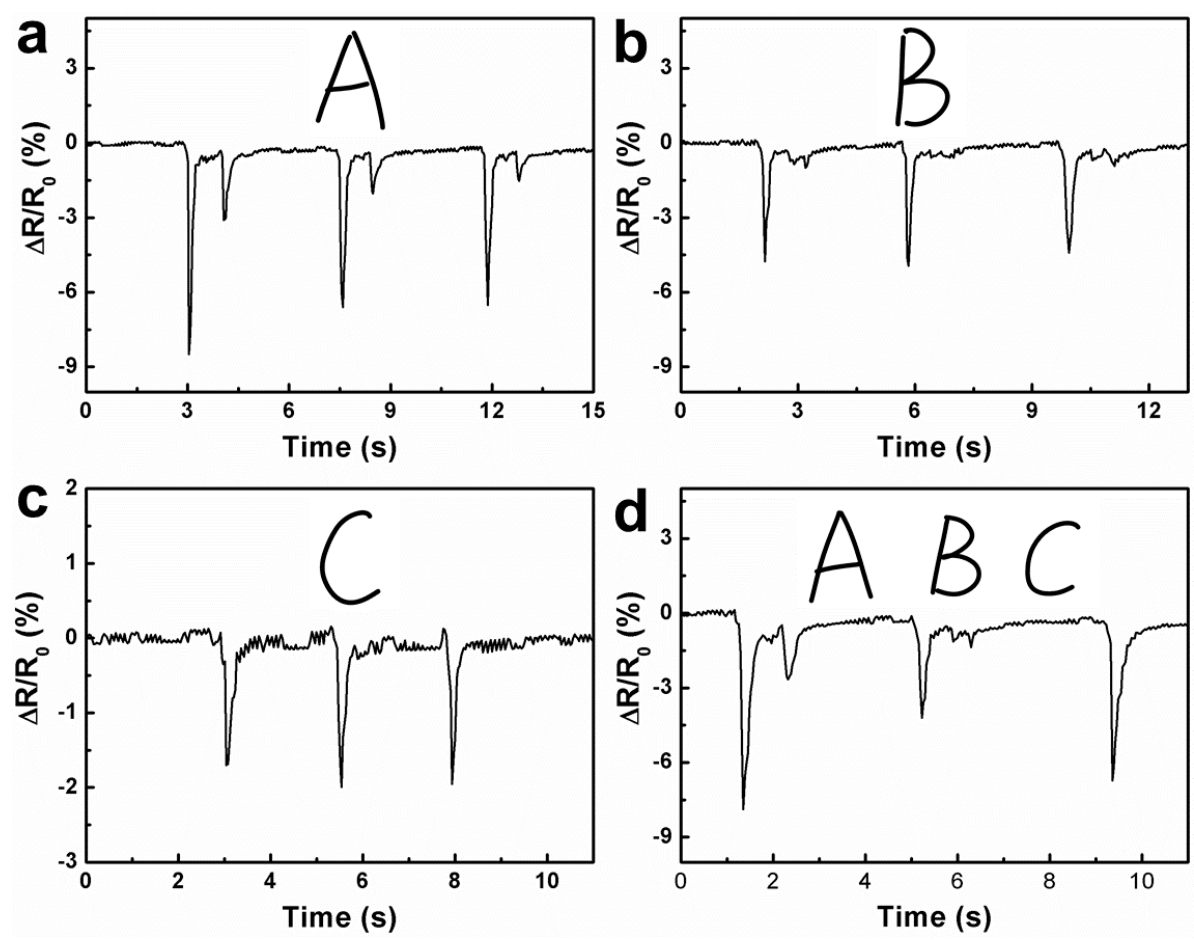

Figure S20. Electrical response of the writing on the pad when written letter "A" (a), "B" (b), "C" (c) and continuous "ABC" (d). Device was fabricated by PHEA-PAM hydrogel. 


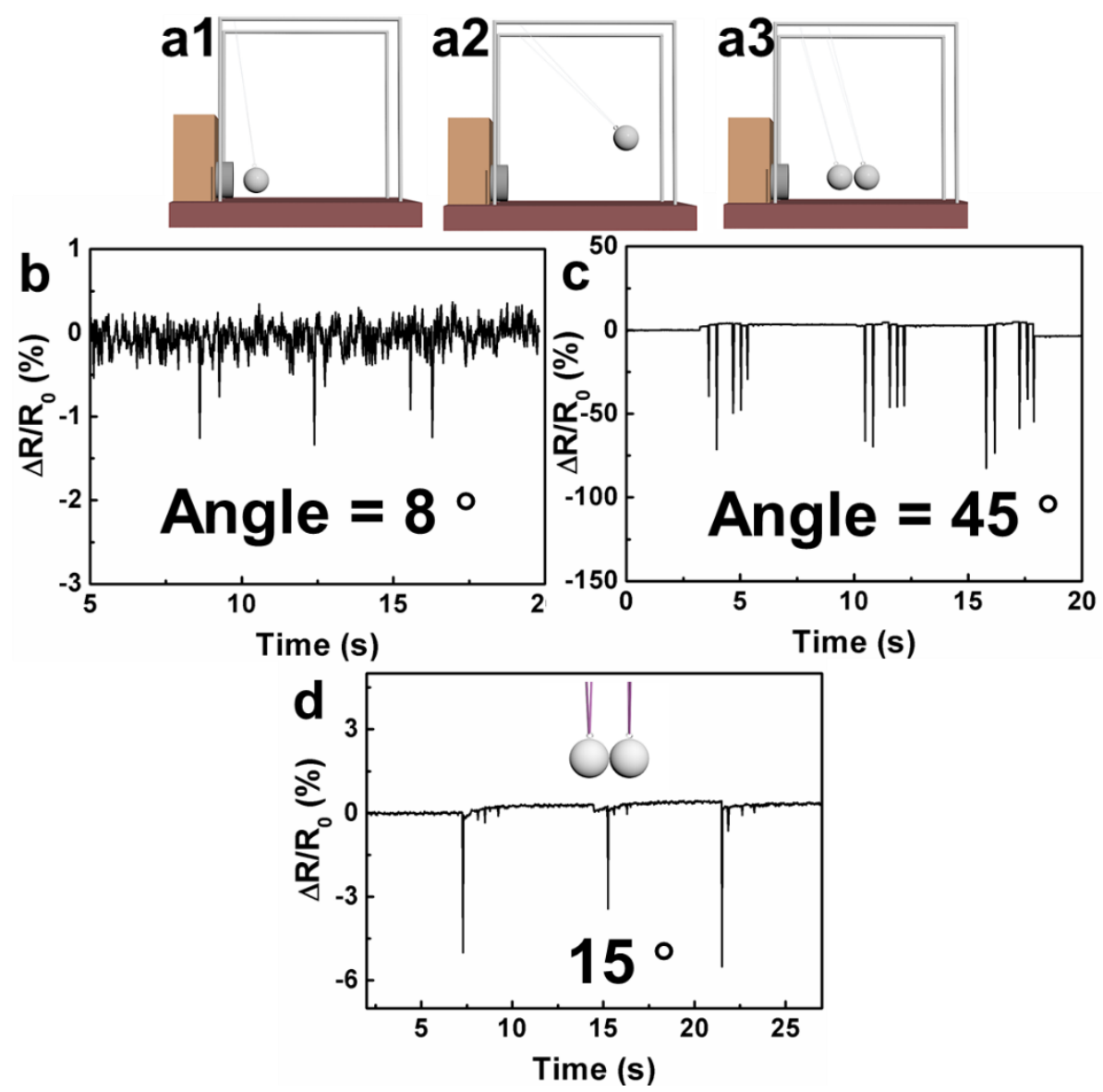

Figure S21. (a1)-(a4) Schematic illustration for the setup to monitor the impact from Newton's cradle. In Newton's cradle, different number of balls (a1 and a2: one, a3: two) were lifted at different angles (a1: $8^{\circ}$, a2: $\left.45^{\circ}\right)$ to hit the LM-Foam to generate the electrical signals; (b) and (c) Curves of resistance change of single ball impact lifting at different angles; (d) Curves of resistance change of impact from two balls lifted at fixed angle of $15^{\circ}$. 
Illustration for the fitted curve $\left(\Delta R / R_{0}\right.$ vs velocity of truck) during truck collision.

The impact tests were conducted at different velocities of truck over 20 times to fit curves. Before collision, the glass ball has the same velocity as truck. Once the collision happens, glass ball will roll front to hit LM-Foam. The friction will be the only force to slow down the ball before collision. The peak value of the signal was corresponded with the maximum compressive strain of the LM-Foam, which was also depended on velocity of the ball before collision. The velocity before collision was calculated by Impulse-Momentum Theorem Formula as shown in equation S1 and S2. The fitted equation was $y=22.5-30.4 x$. The curve was not fitted well at low velocity, which can be explained by

Equation S1: $f t=m v_{t}-m v_{0}$

Equation S2: $v_{t}=\frac{v_{0}-f t}{m}$

$f$ - friction;

$m$ - mass of the ball;

$v_{t}$ - ball velocity before hit on LM-Foam;

$v_{0}$ - Final truck velocity before collision, should be the same as the starting velocity of the ball rolling front;

$t$ - time spent from truck collision to the hit of the ball on LM-Foam.

At low velocity, it cost more time for the ball to roll forth to hit the LM-Foam, resulting in high velocity loss. As a result, the electrical signal was small and did not change too much. While at high velocity, the final velocity was slightly influenced by 
friction and almost the same $\left(v_{t} \approx v_{0}\right)$ with the velocity before the truck stopped due to the short time before collision. Therefore, the resistance change was linear with velocity. The appearance of two stages provides LM-Foam an opportunity for setting up a warning velocity to monitor the collision of production during transportation.

Video S1. The process of foaming. The LM-Foam could be obtained in 2 minutes after $15 \mathrm{~s}$ stirring of the initial mixture (MDI, PTTO, LM and DCM). The video was speeded up for three times.

Video S2. The process of applying external force to LM-Foam-30\% to show the disconnection and connection of circuit.

Video S3. Resistive change of the pad under different writing path at the same direction (Top, Middle and Bottom). The writing pad was fabricated with LM-Foam-40\%. 